\title{
Does Preoperative Weight Loss Predict Significant Postoperative Weight Loss Among Patients who Underwent Laparoscopic Sleeve Gastrectomy?
}

\author{
Ugochukwu Chinaka $^{1}$, Joshua Fultang ${ }^{1}$, Abdulmajid Ali ${ }^{1}$ \\ 1. General Surgery, University Hospital Ayr/University of West of Scotland, Ayr, GBR
}

Corresponding author: Abdulmajid Ali, abdulmajidali@hotmail.com

\section{Abstract \\ Background}

Some bariatric practices, mainly those conducted under public-funded services, have adopted achieving a target preoperative weight loss (PrWL) before offering surgery. There are varied opinions on the correlation between preoperative and postoperative weight loss (PoWL) for the different surgical options. This study investigated the impact of target PrWL on PoWL for patients who underwent laparoscopic sleeve gastrectomy (LSG).

\section{Materials and methods}

A longitudinal retrospective cohort study was carried out on patients who had documented preoperative weight before LSG $(n=155)$ from the authors' institution between February 2008 to October 2017. Patients were grouped into two cohorts based on meeting the 5\% target PrWL or not. The endpoint included percent postoperative weight loss (\% PoWL) at one year and two to three years.

\section{Results}

A total of 155 individuals were identified and analysed. Of these patients, $78.7 \%$ of them $(n=122)$ achieved the $5 \%$ target PrWL (target group) while $21.3 \%(n=33)$ did not (non-target group). At one year, there was no statistical significant difference in the mean \% PoWL between the non-target and target groups $(22.3 \pm 8.1 \%$ versus $19.4 \pm 11.8 \% \mathrm{p}$ value $=0.08$ ). A similar observation was made at two-three years, where the mean $\%$ PoWL in the non-target group was $14.7 \pm 10.7 \%$ versus $16.3 \pm 14.4 \%$ in the target group ( $p$ value $=0.07$ ). Our further analysis highlighted a statistically significant weak inversely proportional correlation between $\%$ PrWL and \% PoWL at one year and two to three years.

\section{Conclusion}

Meeting target PrWL does not significantly impact on PoWL after LSG. Therefore, it should not serve as exclusion criteria for eligible patients who are in need of surgery.

Received 09/14/2019

Review began 09/19/2019

Review ended 10/08/2019

Published 10/09/2019

๑) Copyright 2019

Chinaka et al. This is an open access article distributed under the terms of the Creative Commons Attribution License CC-BY 3.0., which permits unrestricted use, distribution, and reproduction in any medium, provided the original author and source are credited.
Categories: Endocrinology/Diabetes/Metabolism, Gastroenterology, General Surgery

Keywords: preoperative weight loss, bariatric surgery, sleeve gastrectomy, target preoperative weight loss, postoperative weight loss

\section{Introduction}

The stark epidemic proportion of obesity is a reality in today's world [1]. The World Health Organization (WHO) in 2014 revealed estimates that show a staggering 39\% of adults to be overweight and 13\% obese [2]. Obesity is associated with comorbidities such as metabolic disease (such as type 2 diabetes), cardiovascular and joint disease, certain types of cancer, reduction in self-reported quality of life, and increased mortality in the long run [3]. It poses a huge health burden on the individual and has a large societal strain in terms of costs associated with human resources, administration, and long-term patient management [4].

Surgical intervention has been shown to result in greater improvement in terms of weight loss and obesityassociated comorbidities when compared with non-surgical interventions, regardless of the procedure [5]. This epidemic proportion of obesity has witnessed an attendant increase in laparoscopic bariatric surgery worldwide with estimates of about 468,609 procedures performed as of 2013. The most significant rise was that of laparoscopic sleeve gastrectomy (LSG) from 0 to 37\% of the world total from 2003 to 2013 [6].

The criteria set out by the National Institutes of Health (NIH) Consensus Development Conference panel for patients requiring gastric restriction or bypass procedures include well-informed and motivated patients with acceptable operative risks, body mass index (BMI) exceeding 40 or between 35 and 40 with comorbidities (such as severe sleep apnoea, diabetes mellitus) and obesity-induced physical problems. It 
Target PrWL is often encouraged amongst bariatric practitioners mainly public service funded services, before undergoing bariatric surgery to improve patient compliance and outcomes [8-10]. However, the impact of PrWL on a postoperative outcome such as postoperative weight loss (PoWL) has remained controversial [11]. Some have challenged the requirement and efficacy of a preoperative target weight loss before undergoing bariatric procedure [12].

Therefore, there is still a need to further investigate the significance of PrWL on PoWL, especially in patients who underwent LSG.

\section{Materials And Methods}

This study utilised de-identified data from our bariatric database of patients at the authors' institution.

\section{Cohort selection}

We identified all patients who underwent any bariatric operation (gastric band, Roux-en-Y gastric bypass, sleeve gastrectomy, or revisional surgery) $(n=339)$ from February 2008 to October 2017. A total of 166 LSG were performed (included 11 revisions from gastric bands to LSG); however, follow up data for 155 patients were obtained and those without documented postoperative weight after one year excluded from the analysis. Patients were classified as "target" and "non-target" categories based on meeting the 5\% target PrWL or not, and the two groups were compared.

\section{Variables}

We focused on two variables. Percent PrWL (\% PrWL) was determined by subtracting the weight at surgery from the recorded weight at referral to the bariatric service (initial weight) divided by initial weight multiplied by 100. Percent PoWL (\% PoWL) was defined as the difference of the post-surgery weight at one, or two-three years from weight at surgery over the initial weight and expressed as a percentage.

\section{Outcome measure}

Our primary outcome was \% PoWL at one-year and two-three years follow-up. At our institution, we follow up postoperative weights three months to 24 months postoperatively.

The Pearson correlation coefficient ( $r$ ) was used to measure the relationship between both variables. F-test was used to determine the overall statistical significance of this relationship. All data analysis was performed with Microsoft Excel 2013.

\section{Results}

Data from 155 patients was analysed. The median age was 50 years and there were more female than male patients. The average BMI at referral to the bariatric services (start BMI) was $48.5 \mathrm{Kgm}^{-2}$. The average BMI at surgery was $43.8 \mathrm{Kgm}^{-2}$ reflecting an average net weight loss preoperatively from an average of $135.1 \mathrm{Kg}$ to $119.7 \mathrm{Kg}$ at surgery. As seen in Figure 1, of the 155 patients, $78.7 \%$ of patients ( $\mathrm{n}=122)$ achieved the $5 \%$ PrWL (target) while $21.3 \%(n=33)$ did not (non-target). The youngest subject was 26 years and the oldest 62 years at the time of surgery. 


\section{Cureus}

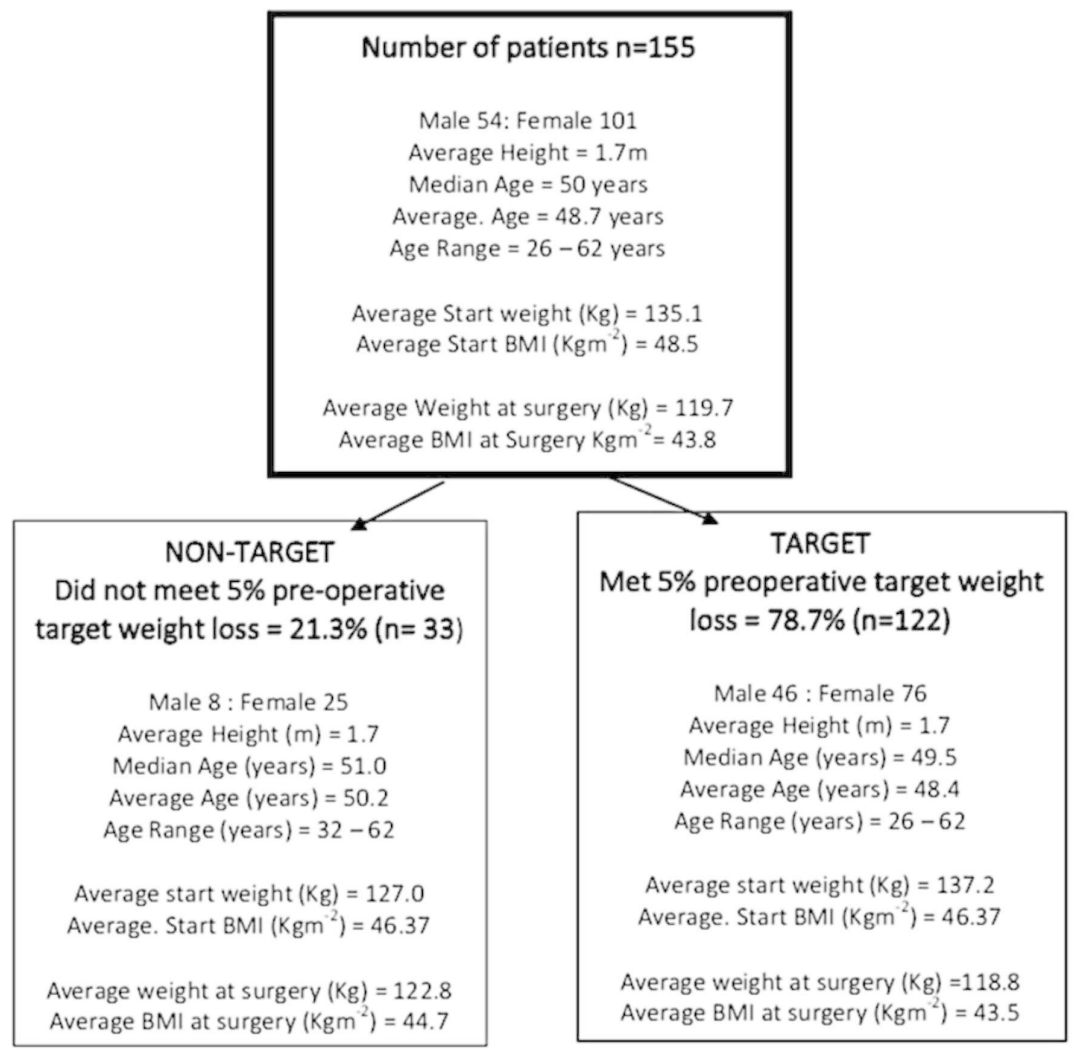

\section{FIGURE 1: Number of patients and division into two cohorts based on meeting/not meeting the $5 \%$ PrWL target}

Both cohorts were comparable in terms of weight and body mass index (BMI) at referral and surgery. The upper limit of the subject's age was similar but the youngest patient in the cohort who did not meet the $5 \%$ PrWL target was 32-years-old, as compared to the 26-years old-in the other cohort.

PrWL: preoperative weight loss.

At one year, patients who didn’t meet $5 \%$ target PrWL had an average \% PoWL of $22.3 \pm 8.1 \%$ versus 19.4 $\pm 11.8 \%$ for those who met $5 \%$ target PrWL ( $p$ value $=0.08$ ) (Figure $2 A$ ).

At two to three years, the average \% PoWL for patients who did not meet the 5\% target PrWL was 14.7 $\pm 10.7 \%$ (Figure $2 B)$. This was slightly lower than the average \% PoWL $(16.3 \pm 14.4 \%)$ recorded in the cohort of subjects who met the $5 \%$ PrWL target ( $\mathrm{p}$ value $=0.07$ ).

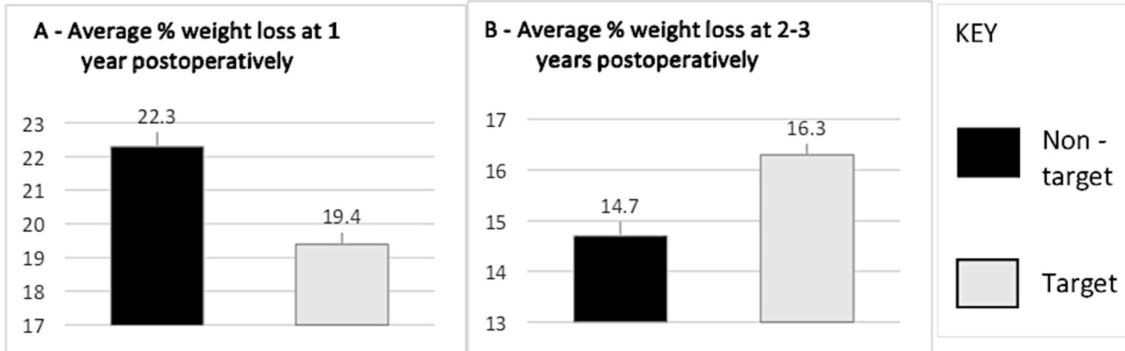

FIGURE 2: Average percentage of weight loss at one year (A) and two to three years (B) postoperatively

At one year postoperatively, non-target group \% PoWL was $22.3 \pm 8.1 \%(n=92)$ vs target $19.4 \pm 11.8 \%(n=22)$ ( $p$ value $=0.08$ ). At two to three years postoperatively, non-target $\%$ PoWL was $14.7 \pm 10.7 \%(n=55)$ vs target $16.3 \pm 14.4 \%(n=21)(p$ value $=0.07)$

PoWL: postoperative weight loss. 


\section{Cureus}

In the target group, the correlation between PrWL and PoWL at one and two to three years are detailed in Figures 3-4.

Target: 1 year postoperatively

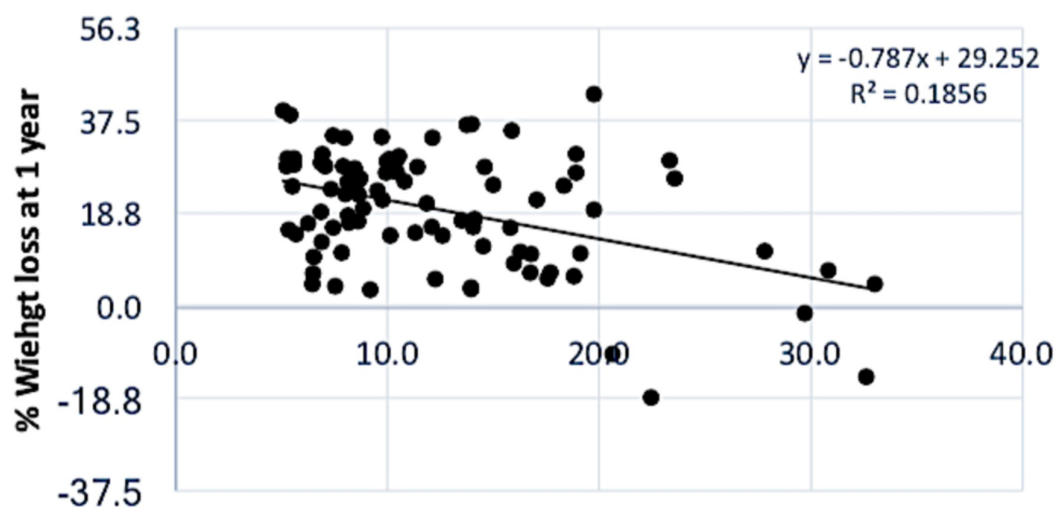

\% Preoperative Weight Loss

FIGURE 3: Graph showing correlation between \% PrWL and \% PoWL at one year in the cohort who met the $5 \%$ preoperative weight loss target Weak inversely proportional relationship in the target group at one year postoperatively. PrWL: preoperative weight loss; PoWL: postoperative weight loss.

\section{Target: 2-3 years postoperatively}

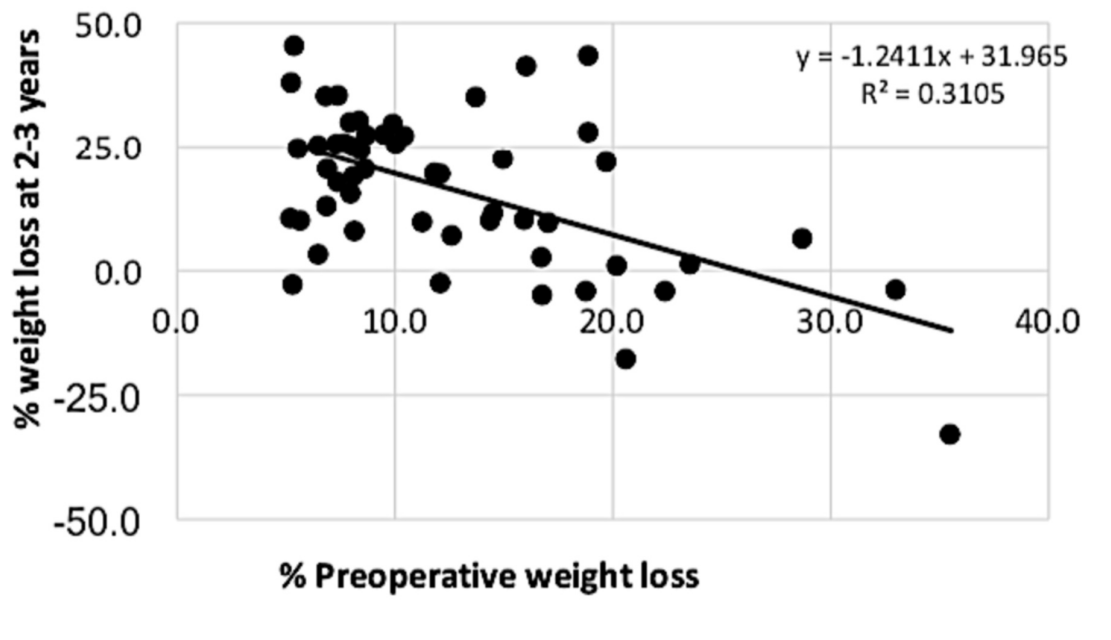

FIGURE 4: Graph showing correlation between \% PrWL and \% PoWL at two to three years for patients who met the $5 \%$ preoperative weight loss target

At two to three years, there was a maintained weak inversely proportional relationship between \% PrWL and $\%$ PoWL.

PrWL: preoperative weight loss; PoWL: postoperative weight loss. 


\section{Cureus}

In the non-target group of patients, a similar relationship between both parameters was observed (Figures 5-6).

\section{Non-Target: 1 year postoperatively}

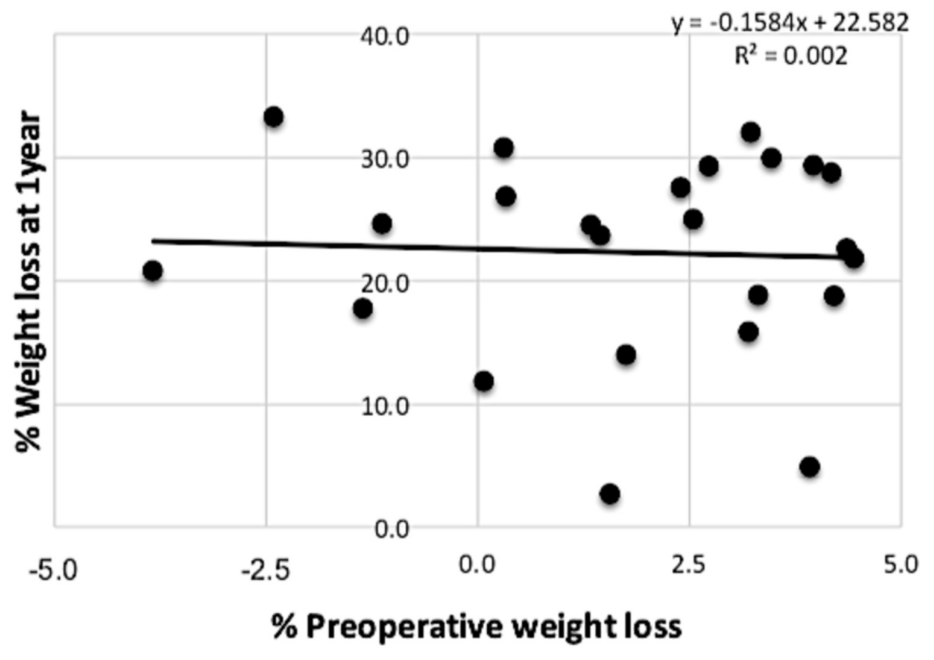

FIGURE 5: Graph showing correlation between \% PrWL and \% PoWL at one year for patients who did not meet the $5 \%$ preoperative weight loss target

There was no correlation between \% PrWL and \% PoWL at one year postoperatively with $r$ values closer to zero.

PrWL: preoperative weight loss; PoWL: postoperative weight loss.

\section{Non-Target: 2-3 years postoperatively}

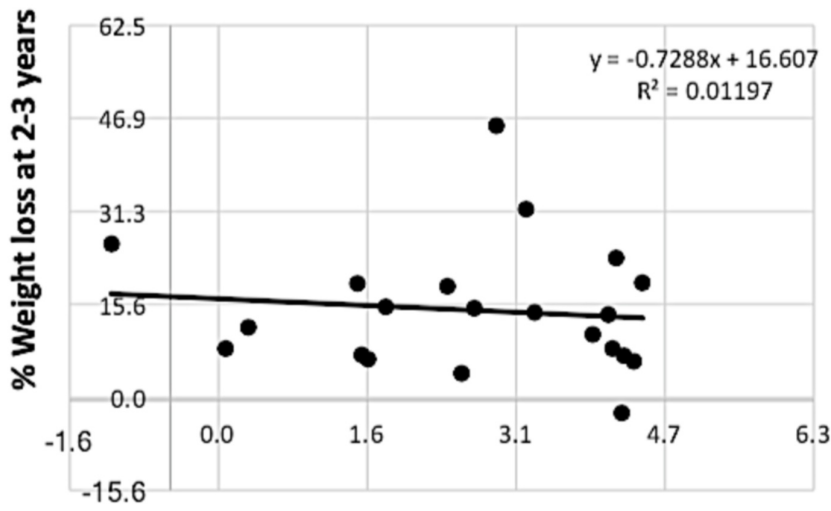

$\%$ Preoperative weight loss

FIGURE 6: Graph showing correlation between \% PrWL and \% PoWL at 


\section{Cureus}

two to three years for patients who did not meet the $5 \%$ preoperative weight loss target

The correlation observed in the non-target group at one year was maintained at two to three years.

PrWL: preoperative weight loss; PoWL: postoperative weight loss.

Analysing combined data from all patients who underwent an LSG is depicted in Figures 7-8.

\section{\% Preoperative weight loss Vs \% Postoperative weight at 1 year for all LSG patients}

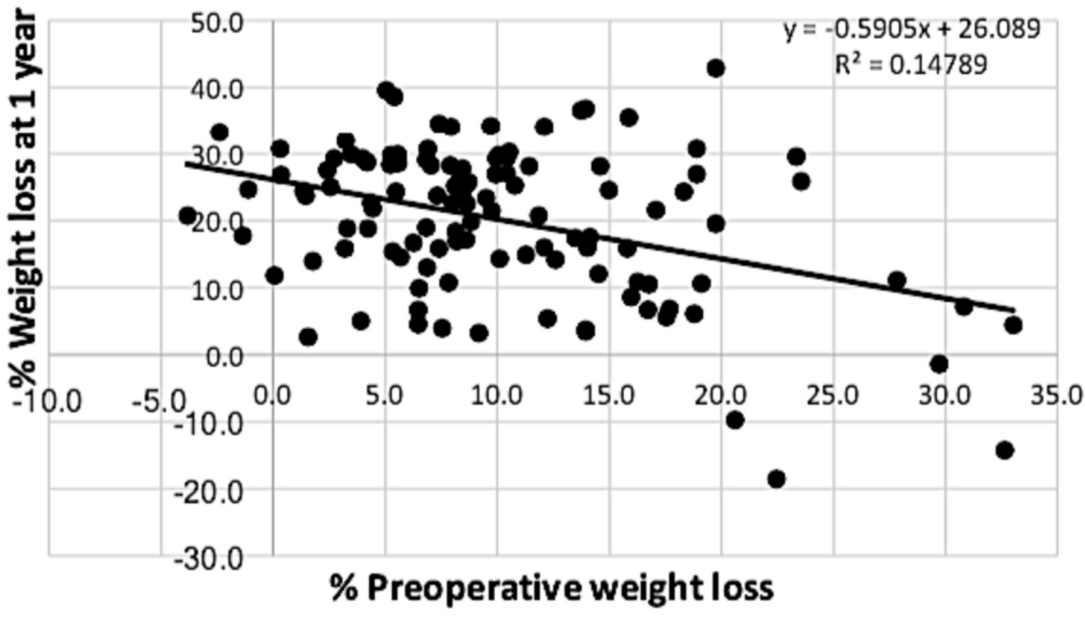

FIGURE 7: Graph showing correlation between \% PrWL and \% PoWL at one year for all laparoscopic sleeve gastrectomy (LSG) patients

Analysing the correlation between \% PrWL and \% PoWL for all patients involved in the study showed a significant weak inversely proportional relationship at one year postoperatively ( $p$ value $<0.001$ ).

PrWL: preoperative weight loss; PoWL: postoperative weight loss.

\section{\% Preoperative weight loss Vs \% Postoperative weight at}

\section{2-3 years for all LSG patients}

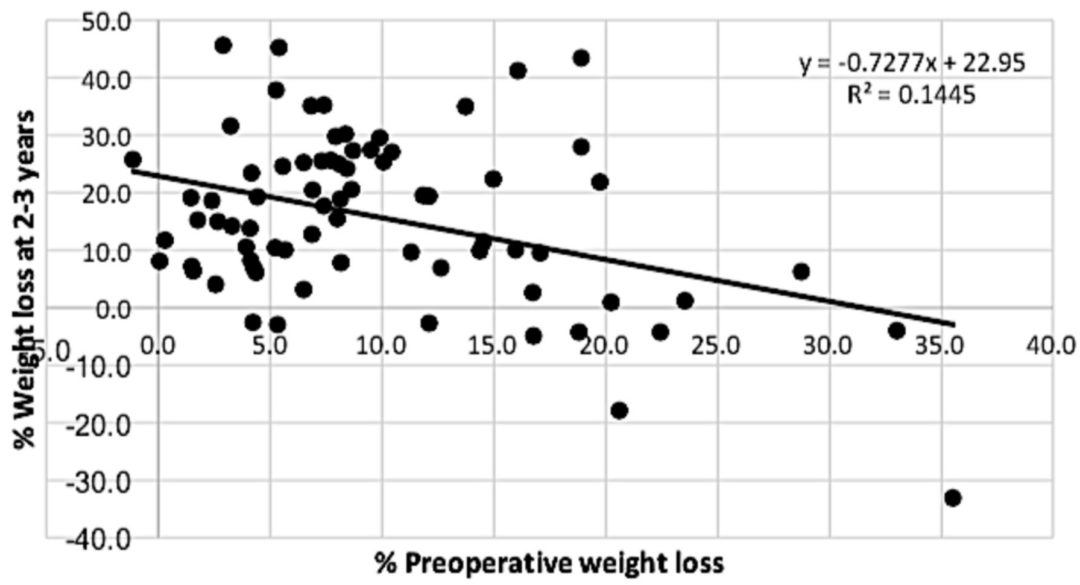


FIGURE 8: Graph showing correlation between \% PrWL and \% PoWL at two to three years for all laparoscopic sleeve gastrectomy (LSG) patients

This statistically weak inversely proportional relationship was maintained at two to three years postoperatively ( $p$ value $<0.001$ ).

PrWL: preoperative weight loss; PoWL: postoperative weight loss.

\section{Discussion}

Bariatric (weight loss) surgery as at today is safe, effective in producing significant sustainable weight loss, and leads to the improvement or resolution of co-morbidities associated with obesity [13-14]. LSG as a bariatric option has rapidly gained popularity and is considered the second most performed bariatric procedure in the world [15]. Despite the increased acceptance of weight loss surgery, less than $1 \%$ of eligible candidates end up receiving surgery in some climes [16]. In undertaking bariatric services, some institutions have adopted a policy of target PrWL citing advantages such as reduced peri-operative morbidity, decreased operating time with less blood loss, and possible motivation for further weight loss [17].

At the authors' institution, the majority of the patients participated in the hospital's preoperative program and are expected to achieve a target weight loss of $5 \%$ before undergoing surgery. However, some who did not meet the target weight loss but reasonably fulfilled the NIH Consensus Development Conference and the Scottish National Planning Forum (NPF) guidelines after bariatric multidisciplinary team (MDT) review, were offered surgery. We sought to determine whether target PrWL impacted significantly on PoWL. Our study observed no strong correlation between PrWL and PoWL amongst the patients that had LSG at one and twothree years postoperative years.

In a meta-analysis done in 2011 involving 17 trials and 10 studies by Cassie et al. and a most recent one in 2014 by Gerber et al. (included 23 publications and two review articles), the authors were inconclusive about the effect of PrWL as a result of the heterogeneity in the various study designs [3,9].

In a single-center review of 192 patients who underwent LSG during a nine-month study period, Parmar et al. reported no correlation between those who lost 'high' or 'low' preoperative weight (based on comparison to the median percent PrWL of 5.1\%) and postoperative weight at one year [18].

Another study by Sherman et al. in their review of 141 patients also identified that PrWL is not a reliable predictor of PoWL [11]. Watanabe A et al. in their work noted that the extent of PrWL did not contribute to better weight loss during the overall period [15]. Our findings further align with the above studies that target PrWL does not significantly impact on PoWL after LSG.

We recognise the limitation of this study given its single institutional non-randomised review nature, which may raise the possibility of selection bias and may not entirely project the broader bariatric population.

\section{Conclusions}

Our study observed that achieving target PrWL does not significantly impact PoWL as reported in previous studies. Eligible bariatric candidates should not be denied surgery based on target PrWL. We recommend further prospective trials to delineate the impact of PrWL on LSG outcomes.

\section{Additional Information}

\section{Disclosures}

Human subjects: All authors have confirmed that this study did not involve human participants or tissue. Animal subjects: All authors have confirmed that this study did not involve animal subjects or tissue. Conflicts of interest: In compliance with the ICMJE uniform disclosure form, all authors declare the following: Payment/services info: All authors have declared that no financial support was received from any organization for the submitted work. Financial relationships: All authors have declared that they have no financial relationships at present or within the previous three years with any organizations that might have an interest in the submitted work. Other relationships: All authors have declared that there are no other relationships or activities that could appear to have influenced the submitted work.

\section{References}

1. Friedrich MJ: Global obesity epidemic worsening. JAMA. 2017, 318:603. 10.1001/jama.2017.10693

2. WHO obesity. (2018). Accessed: September 1, 2019: https://www.who.int/topics/obesity/en/.

3. P Gerber, C Anderin, A Thorell: Weight loss prior to bariatric surgery: an updated review of the literature . 
Scand J Surg. 2015, 104:33-39. 10.1177/1457496914553149

4. Tremmel M, Gerdtham UG, Nilsson PM, Saha S: Economic burden of obesity: a systematic literature review . Int J Environ Res Public Health. 2017, 14:435. 10.3390/ijerph14040435

5. Colquitt JL, Pickett K, Loveman E, et al.: Surgery for weight loss in adults . Cochrane Database Syst Rev. 2014, 8:3641. 10.1002/14651858.CD003641.pub4.

6. Angrisani L, Santonicola A, Iovino P, Formisano G, Buchwald H, Scopinaro N: Bariatric surgery worldwide 2013. Obes Surg. 2015, 25:1822-32. 10.1007/s11695-015-1657-z

7. Hubbard VS, Hall WH: Gastrointestinal surgery for severe obesity. Obesity Surgey. 1991, 1:257-265. 10.1381/096089291765560962

8. Hutcheon DA, Hale AL, Ewing JA, et al.: Short term preoperative weight loss and postoperative outcomes in bariatric surgery. J Am Coll Surg. 2018, 226:514-524. 10.1016/j.jamcollsurg.2017.12.032

9. Cassie S, Menezes C, Birch DW, Shi X, Karmali S: Effect of preoperative weight loss in bariatric surgical patients: a systematic review. Surg Obes Relat Dis. 2011, 7:760-767. 10.1016/j.soard.2011.08.011

10. Livhtis M, Mercado C, Yermilov I, et al.: Does weight loss immediately before bariatric surgery improve outcomes: a systematic review. Surg Obes Relat Dis. 2009, 5:713-721. 10.1016/j.soard.2009.08.014

11. Sherman WE, Lane AE, Mangeri CW, Choi YU, Faler BJ: Does preoperative weight change predict postoperative weight loss after laparoscopic sleeve gastrectomy?. Bariatr Surg Pract Patient Care. 2015, 10:126-129. 10.1089/bari.2015.0023

12. Conaty EA, Bonamic NJ, Gitelis ME, et al.: Efficacy of a required preoperative weight loss program for patients undergoing bariatric surgery. J Gastrointest Surg. 2016, 20:667-73. 10.1007/s11605-016-3093-3

13. Mingrone G, Panunzi S, De Gaetano A, et al.: Bariatric surgery versus conventional medical therapy for type 2 diabetes. N Engl J Med. 2012, 366:1577-85. 10.1056/NEJMoa1200111

14. Adams TD, Davidson LE, Litwin SE, et al.: Health benefits of gastric bypass surgery after 6 years . JAMA. 2012, 308:1122-1131. 10.1001/2012.jama.11164

15. Watanabe A, Seki Y, Haruta H, Kikkawa E, Kasama K: Preoperative weight loss and operative outcome after laparoscopic sleeve gastrectomy. Obes Surg. 2017, 27:2521. 10.1007/s11695-017-2697-3

16. Encinosa WE, Bernard DM, Steiner CA, Chen CC: Use and costs of bariatric surgery and prescription weightloss medications. Health Aff. 2005, 24:1039-46. 10.1377/hlthaff.24.4.1039

17. Ali MR, Baucom-Pro S, Broderick-Vila GA, et al.: Weight loss before gastric bypass: feasibility and effect on postoperative weight loss and weight loss maintenance. Surg Obes Relat Dis. 2007, 3:515-20. 10.1016/j.soard.2007.05.002

18. Parmar AD, Drosdeck JM, Mattar SG, Spight D, Husain FA: Impact of preoperative weight loss on postoperative weight loss after sleeve gastrectomy. Bariatr Surg Pract Patient Care. 2018, 13: 69-74. 10.1089/bari.2018.0001 